\title{
THE CAPILLARY PATTERN IN HUMAN MASSETER MUSCLE DURING AGEING
}

\author{
ERIKA CVETKO ${ }^{1}$, JIŘÍ JANÁČEK ${ }^{2}$, LUCIE KUBÍNOVÁ ${ }^{2}$ AND IDA ERŽEN ${ }^{\bowtie, 1}$ \\ ${ }^{1}$ Institute of Anatomy, Faculty of Medicine, University of Ljubljana, Ljubljana, Slovenia; ${ }^{2}$ Department of \\ Biomathematics, Institute of Physiology, Academy of Sciences of the Czech Republic \\ e-mails: erika.cvetko@mf-uni-lj.si; janacek@biomed.cas.cz; kubinova@biomed.cas.cz; ida.erzen@mf.uni-lj.si \\ (Received June 12, 2013; revised August 20, 2013; accepted October 8, 2013)
}

\begin{abstract}
The effect of ageing on the capillary network in skeletal muscles has produced conflicting results in both, human and animals studies. Some of the inconsistencies are due to non-comparable and biased methods that were applied on thin transversal sections, especially in muscles with complicated morphological structures, such as in human masseter muscle. We present a new immunohistochemical method for staining capillaries and muscle fibres in $100 \mu \mathrm{m}$ thick sections as well as novel approach to 3D visualization of capillaries and muscle fibres. Applying confocal microscopy and virtual 3D stereological grids, or tracing capillaries in virtual reality, length of capillaries within a muscle volume or length of capillaries adjacent to muscle fibre per fibre length, fibre surface or fibre volume were evaluated in masseter muscle of young and old subjects by an unbiased approach. Our findings show that anatomic capillarity is well maintained in masseter muscle in old subjects; however, vascular remodelling occurs with age, which could be a response to changed muscle function and age-related muscle fibre type transformations.
\end{abstract}

Keywords: 3D analysis, capillaries, confocal microscopy, human, masseter muscle.

\section{INTRODUCTION}

The effect of ageing on the capillary network in skeletal muscles has been studied in limb muscles predominantly. Both, human and animals studies have produced conflicting results. Some of the inconsistencies are due to noncomparable and biased methods that were applied on thin transversal sections, especially in muscles with complicated morphological structures, e.g. multipennate muscles composed of nonparallel muscle fibres of variable length. Such an anatomy suggests a complex ramification of the capillary network in 3D space which could not be satisfactorily assessed from 2D sections.

Most of the published studies on skeletal muscles counted capillary profiles and presented their results either as the number of capillary profiles per number of muscle fibre profiles $(\mathrm{C} / \mathrm{F})$, per muscle cross-sectional area $(\mathrm{CD})$ or around a fibre profile (CAF), or the number of capillary contacts per fibre (CC) (Degens et al., 1994; Hepple and Vogell, 2004; Croley et al., 2005; Mathieu-Costello et al., 2005; Ryan et al., 2006; Čebašek et al., 2010). Further, in human posterior crycoarythenoideus muscle (PCA) Lyon et al. (2007) estimated the length of capillaries per fibre and separately for each fibre type volume and surface area, using the orientator method. Methods based on Euler number (cf. Nyengaard, 1999; Knust et al., 2009; Wulfson et al., 2009) have successfully been applied in other research fields like lung, kidney etc., however they have not been introduced into the muscle research yet.

Research on animal muscles reported either maintained, unchanged capillary supply (Brown, 1987; Mitchell et al., 1991; Hepple and Vogell, 2004; Mathieu-Costello et al., 2005) or reduced capillary network (Degens et al., 1994) with ageing. Similarly, in human muscles capillary supply has been reported to be either maintained (Denis et al., 1986; Proctor et al., 1995; Lyon et al., 2007) or reduced (Coggan et al., 1992; Frontera et al., 2000; Croley et al., 2005; Ryan et al., 2006).

Compared to limb and trunk muscles masseter and PCA have special characteristics: masseter is active during the most part of the day to perform complex oral function such as mastication, speech, facial expression and postural control of mandible which are maintained with age. Similarly, the activity level was postulated to be the reason that there is a lack of age-related change in neuromuscular junction lengths or in the terminal axonal branching patterns at these junctions within the human PCA (Gambino et al., 1990), which is active during inspiration, expiration and phonation. 
Several studies proved also denser capillarization of type 1 than type 2 muscle fibres (oxidative and glycolytic fibres, respectively). Better capillary supply of type 1 than type 2 fibres was demonstrated in the triceps brachii, vastus lateralis and soleus muscles (Sjøgaard, 1982). Type 1 fibres in the triceps brachii muscle posses on average, 4.85 capillary profiles around each fibre, while in more oxidative soleus this number is 6.89; however, the difference should reflect varying oxidative capacity of the same muscle fibre type within various muscles.

Further, the ratio of capillary profiles to fibres was significantly larger in those muscles that exhibited a significantly larger proportion of oxidative fibre types (Cui et al., 2008), while the number of capillary profiles per muscle cross-sectional area was larger in type 1 muscle fibres than in type 2 muscle fibres; i.e., on average, in the oxidative muscle there are more capillary profiles per fibre compared to glycolytic muscle (Nelissen-Vrancken et al., 1993). Long-term (4 weeks) ischemia increased significantly the capillary-to-fibre ratio in the soleus muscle, composed predominantly of type 1 fibres, whereas capillarity in the more glycolytic portion of the gastrocnemius muscle did not change.

Type 2 fibres of orofacial muscles display a relatively higher activity of oxidative enzymes than those of the limb muscles (Stål et al., 1990), although it has been proved elsewhere that fibre size is decisive for the capillarity and the level of oxidative metabolism is just a secondary effect (Cotter et al., 1973; Brown et al., 1976; Škorjanc et al., 1998; Egginton and Hudlická, 2000; Hepple and Vogel, 2004).

In limb muscles exercise training may prevent the age-related loss of muscle capillaries supplying type 1 fibres, while it is not efficient in maintaining capillary network to type $2 \mathrm{a}$ and $2 \mathrm{~b}$ fibres. This suggests that ageing is accompanied by lower angiogenic potential of type 2 muscle fibres (Proctor et al., 1995). Caution should be adopted when comparing the limb and jaw muscles because of intrinsic anatomic and behavioural differences in the masticatory apparatus and limb function. It could be speculated that postural function is predominant in limb muscle with ageing but not in jaw-closing muscles.

In our previous publication (Čebašek et al., 2010) we proved that counting capillary profiles in $2 \mathrm{D}$ can underestimate capillary length by up to 75 percent in muscles with nonparallel muscle fibres and complicated anatomic composition. To avoid this estimation error we applied the method for estimating characteristics of the capillary network in 3D, introduced in our previous publications (Kubínová et al., 2001; Eržen et al., 2011;
Janáček et al., 2011). The method requires thick tissue sections from which a complete series of optical sections is captured by a confocal microscope. The capillary network is visualized in 3D and analysed within the whole sample volume. Although the method is more time consuming than the 2D method, muscle sample preparation requires a skilful technician and results need to be corrected for shrinkage in the $\mathrm{z}$ direction (Janáček et al., 2012). This method provides a more complete information on the capillary anatomy in the studied muscles. Parameters that can be estimated within a unit of muscle tissue are the capillary length, number of capillary branchings, average capillary length and orientation of capillaries, i.e. the degree of anisotropy. Further, we measure the length of capillaries supplying muscle fibre type 1 and type 2 per muscle fibre length (Lcap/ Lfib), per muscle fibre surface area (Lcap/Sfib) and per muscle fibre volume (Lcap/Vfib).

The aim of the present study was to find out if in human masseter muscle vascular remodelling occurs with age, which could be a response to changed muscle function and age-related muscle fibre type transformations.

\section{MATERIAL AND METHODS}

Samples of masseter muscles were taken at autopsy from eight young subjects (average age $28.4 \mathrm{yrs}$, SEM $=2.7$, range 19-39 yrs) and eight elderly subjects (average age $78.3 \mathrm{yrs}, \mathrm{SEM}=1.5$, range $70-84 \mathrm{yrs}$ ) who passed away either after brain stroke or in car accidents. Most autopsy samples of the super-ficial part of the muscle were excised up to $26 \mathrm{hrs}$ postmortem, one sample $48 \mathrm{hrs}$ and we do not have precise information for one sample. The National Medical Ethics Committee of the Republic of Slovenia approved the research.

After excision muscle samples were immersed in liquid nitrogen and kept frozen in deep freezer at $70^{\circ} \mathrm{C}$. Transversal sections $100 \mu \mathrm{m}$ thick were cut with the cryotome Cryo-Star HM 560 (Microm International $\mathrm{GmbH}$, Waldorf, Germany) into cold phosphate buffered saline. They were immediately transferred into cold fixative for $48 \mathrm{hrs}$. Both solutions were kept at a constant temperature of $+4^{\circ} \mathrm{C}$ on a mixture of water and ice.

The fixative contained $10 \%$ formaldehyde and $0.1 \%$ glutaraldehyde in $5 \mathrm{mM}$ Tris buffer with $0.5 \mathrm{mM}$ EDTA and proteinase-K $0.2 \%$. Throughout the fixation, rinsing and staining procedures sections were kept in small homemade baskets, which were gently transferred from one solution to the other. The penetration of all media and antibodies was enabled from all directions and at 
the same time the sections were protected from mechanical injury.

\section{IMMUNOHISTOCHEMISTRY}

All antibodies were diluted in $2 \%$ bovine serum in phosphate buffered saline at $\mathrm{pH} 7.2$ to 7.4. After thorough rinsing in phosphate buffered saline with Triton X-100 (PBST) $20 \mathrm{mM}$, sections were immersed in $20 \%$ normal goat serum for two hours followed by an overnight incubation in F8 (1:1000 to 1:2000) (Dako, Glostrup, Denmark), rinsing in PBST (1-2 hrs), incubation in anti mouse Alexa-Fluor 488 (1:500) (Invitrogen) for two hours, rinsing in PBST for 1-2 hrs, overnight incubation in monoclonal collagen $(1: 150)$ (Dako, Glostrup, Denmark), rinsing in PBST for 1-2 hrs, anti mouse Alexa-Fluor 546 1:500, rinsing in PBST (1-2 hours), overnight incubation in fluorescein conjugated Ulex europaeus agglutinin I (UEA) (Sigma Aldrich, St Louis, USA) 1:500 and final thorough rinsing in PBST for one hour.

Finally, sections were carefully transferred to the slides and embedded in Prolong ${ }^{\circledR}$ Gold antifade (Invitrogen). To prevent tissue deformation the distance between the slide and the cover slip was maintained by two layers made by the ImgEdge pen (Dako, Glostrup, Denmark) (see Janáček et al., 2011).

\section{CONFOCAL MICROSCOPY}

Completely registered stacks of images of the whole thickness of specimen including its upper and lower neighbourhood were captured from thick sections by LSM 510 confocal microscope (Karl Zeiss, Jena, Germany) using a Plan Apochromat $20 \times(\mathrm{NA}=0.75)$ dry objective. Green and red fluorescence was excited with argon $(488 \mathrm{~nm})$ and $\mathrm{He} / \mathrm{Ne}(543 \mathrm{~nm})$ lasers, respectively. Emission signal was filtered using a narrow band $(505-530 \mathrm{~nm})$ and an LP 560-nm filter. Images $(512 \times 512$ pixels $)$ were captured at a pixel size from 0.62 to $1 \mu \mathrm{m}$ with intervals $1 \mu \mathrm{m}$ in axial direction.

\section{IMAGE PROCESSING}

\section{Correction of the tissue shrinkage}

The apparent thickness of the confocal stack may be strongly influenced by physical deformation of the sample (Dorph-Petersen et al., 2001) and optical phenomena (Hell et al., 1993). The deformation occurs even in well fixed skeletal muscle samples; it is spatially homogeneous and can be corrected by adapting the intersection axial distance properly (Janáček et al., 2012).
The apparent thickness of the transversal sections was estimated as the distance between optical sections where the average intensity at the section drops at half of the maximal value of section average intensity (the parameter is usually called FWHM - full width at half maximum).

The axial calibration of individual stacks was multiplied by a correction factor to match the apparent distance of the lower and upper surface of the thick section to the original thickness known from the microtome setting. The precision of the microtome calibration was carefully checked by authorized service.

The apparent thickness of the transversal sections was estimated as the distance between extreme optical sections where the average intensity at the section drops at half of the maximal value of section average intensity (the parameter is usually called FWHM full width at half maximum).

\section{Automatic detection of capillaries}

The green channel of the 3D image coded by 256 levels of gray was pre-processed to enable automatic segmentation by thresholding. The image was smoothed by 3D Gaussian filter with parameter sigma set to $2 \mu \mathrm{m}$ in order to decrease level of the noise. Then the slowly varying background due to nonspecific staining was removed by subtracting the lower Lipschitz envelope with slope $1 \mu \mathrm{m}^{-1}$ (Štencel and Janáček, 2006). Binary image of capillaries obtained by thresholding was thinned by procedure called skeletonization using the 6-pass algorithm (Palágyi and Kuba, 1998) yielding line binary skeleton. The binary image then converted to spatial geometric graph of capillaries, composed of straight line segments (the graph edges) connected at endpoints (the graph vertices) by generating chains of line segments during automatic tracing of the thin lines in the binary image between the endpoints or branching points and connecting them to the centres of mass of remained thick parts. The graph was then resampled to obtain the uniform length of the line segments equal to $5 \mu \mathrm{m}$, because the chains obtained by tracing procedure composed of many short segments with irregular course do not represent well the smooth course of capillaries and would yield overestimates of length. The image processing and further measurements were accomplished by dedicated plug-ins of Ellipse programme (ViDiTo, Slovakia).

\section{Manual tracing of capillaries}

The graphs of capillaries were corrected manually using 3D editing program. We used a custom made programme with desktop virtual reality interface for PC with MS Windows (Microsoft, USA). The interface 
consists of stereoscopic volume rendering of images with 3D cursor controlled by a haptic device (SensableOmni, SenseGraphics AB, Sweden) with force proportional to the gradient of the image intensity. The haptic feedback serves as a cue for depth perception and enables better placement of cursor in the capillaries. The stereoscopic rendering is accomplished either by a special graphic card with dedicated monitor and LCD shutter glasses or by anaglyph visualization with a common display and colour (RB) glasses. The program enables 3D drawing of capillaries as chains of line segments, moving individual points, connecting the chains together and deleting the vertices or chains. Eventually other 3D editor as Filament editor in Amira (VSG) or Tracer plug-in Ellipse may be used for editing.

\section{Assigning capillaries to muscle fibres}

Muscle fibre outlines were manually drawn at two to four levels within the stack from the topmost to the bottom image inside the sample. The outlines were used for muscle fibre diameter estimation and for assignment of the capillaries to individual fibres (see Janáček et al., 2009). Fibre diameter was defined as the length of projection ('single' Feret's diameter) in the direction that is perpendicular to the direction of the maximal length of projection, implying that the diameter defined in this way is less influenced by the angle of cutting.

The neighbourhood of the fibre was represented by triangulated surface spanning contours obtained by dilation of contours of the fibre cross-section within the sample by $15 \mu \mathrm{m}$. The triangulated surface was selected by a dynamic programming algorithm from triangulated surfaces spanning the contours as the surface encompassing maximal volume. The total length of the capillaries around each individual muscle fibre was calculated by summing up the lengths of the line segments inside the fibre neighbourhood. Capillaries were assigned to a fibre when they were present within the $15-$ $\mu \mathrm{m}$ neighbourhood from the fibre. Several capillaries could be assigned to two or more fibres. The calculation was implemented in CapFibres plug-in of Ellipse program.

\section{D visualisation of capillaries and muscle fibres}

The surfaces of muscle fibres were modelled by triangulated surfaces spanning the outlines of the fibres extremal cross-sections. The capillaries were modelled by chains of cylinders with constant diameter joined by spheres with the same diameter. The capillaries and fibre surfaces were rendered in Contours plug-in of program Ellipse using OpenGL graphic library (SGI, USA).

\section{Parameters describing the capillary pattern in muscle samples}

The length of capillaries is calculated by summing the line segments lengths. The course of capillaries and their distribution in regard to muscle fibre orientation were described by tortuosity, connectivity (i.e. number of branchings) and the degree of anisotropy (orientation).

We define tortuosity as average curvature - the sum of angles (in radians) between successive line segments divided by the total capillary length.

We estimated connectivity by the number of capillary branchings counted manually from $3 \mathrm{D}$ visualisation of capillaries. The number is equal to estimate of $-2 \chi(\chi$ is Euler-Poincaré characteristic Gundersen et al., 1993) for samples of capillary network, i.e. connected network without endpoints and with branching points where exactly three chains connect.

Orientation of capillaries was judged by the degree of anisotropy computed from eigenvalues of mean structural tensor in the following way: Each line segment of the traced capillaries $\langle a, b\rangle$ with end-points $a$ and $b$ of length $L$ and direction $v=(b-a) / L$ yielded the matrix

$$
U=\left(\begin{array}{lll}
v_{1} \cdot v_{1} & v_{1} \cdot v_{2} & v_{1} \cdot v_{3} \\
v_{2} \cdot v_{1} & v_{2} \cdot v_{2} & v_{2} \cdot v_{3} \\
v_{3} \cdot v_{1} & v_{3} \cdot v_{2} & v_{3} \cdot v_{3}
\end{array}\right) .
$$

The average of all matrices (weighted by length) was calculated $T=\sum_{i} L_{i} U_{i} / \sum_{i} L_{i}$.

The anisotropy characteristics were calculated from the eigenvalues $\lambda_{1} \geq \lambda_{2} \geq \lambda_{3} \geq 0$ of the matrix $T$

$$
\frac{2 \lambda_{3}}{\lambda_{1}+\lambda_{2}}
$$

The obtained numbers can be in range from 1 (i.e. isotropy - capillaries express no preferential direction) to infinity (i.e. total anisotropy- all capillaries run in the same direction presumably parallel to the muscle fibre axes).

The mean capillary length was estimated as $2 / 3$ of the total length divided by the number of branchings. The estimator is based on assumption that each capillary is connected to 2 other capillaries at each of both ends. 


\section{Parameters describing capillary supply of muscle fibre types}

We estimated capillary characteristics: length of capillaries per muscle fibre length (Lcap/Lfib), per muscle fibre surface area (Lcap/Sfib) and per muscle fibre volume (Lcap/Vfib) of type 1 and type 2 muscle fibres separately. Muscle fibre types were selected by their diameter based on our previous research on masseter muscle (see Cvetko et al., 2012). In most samples fibres smaller than $30 \mu \mathrm{m}$ were classified as type 2 fibres and fibres larger than $40 \mu \mathrm{m}$ as type 1 fibres. Since in three out of 16 samples muscle fibres were much smaller, those smaller than $20 \mu \mathrm{m}$ were taken as type 2 fibres and those larger than $30 \mu \mathrm{m}$ as type 1 fibres. All the remaining fibres were hybrid fibres (between type 1 and type 2 fibres) - they were excluded from the study.

\section{STATISTICS}

The statistical package Systat Version 5 was applied to calculate mean values and standard error of the mean (SEM) for the length of capillaries per muscle volume (Lcap/Vmuscle), Lcap/Lfib, Lcap/Sfib, Lcap/Vfib, tortuosity, anisotropy, Lbr, Nbr/Vmuscle and fibre diameter. Differences in mean values of the above para- meters between masseter muscles of young and old subjects and between muscle fibres of type 1 and 2 were tested with independent samples test t. Differences among means were considered statistically significant, if $\mathrm{p}<0.05$.

\section{RESULTS}

The triple immunohistochemical staining detected capillaries in yellow - green and muscle fibres in red in most young and old subjects (Fig. 1). In several muscle samples, however, UEA lectin failed to detect capillaries. Those samples were excluded from the study.

Fig. 2 shows 3D rendering of capillaries and muscle fibres in masseter muscle of young and old subjects.

The characteristics of the capillary network were quite variable within every age group (Table 1). We could not detect any significant differences among both age groups; however, there was a trend toward reduction in curvature and branch density in the old group with a consequent elongation of the average capillary branch length. We need to point out that values for Lcap/Vmuscle and $\mathrm{Nbr} / \mathrm{Vmuscle}$ could be biased as due to ethic reasons, we could not measure total volume of masseter muscles. The latter could therefore lead to a possible reference trap.
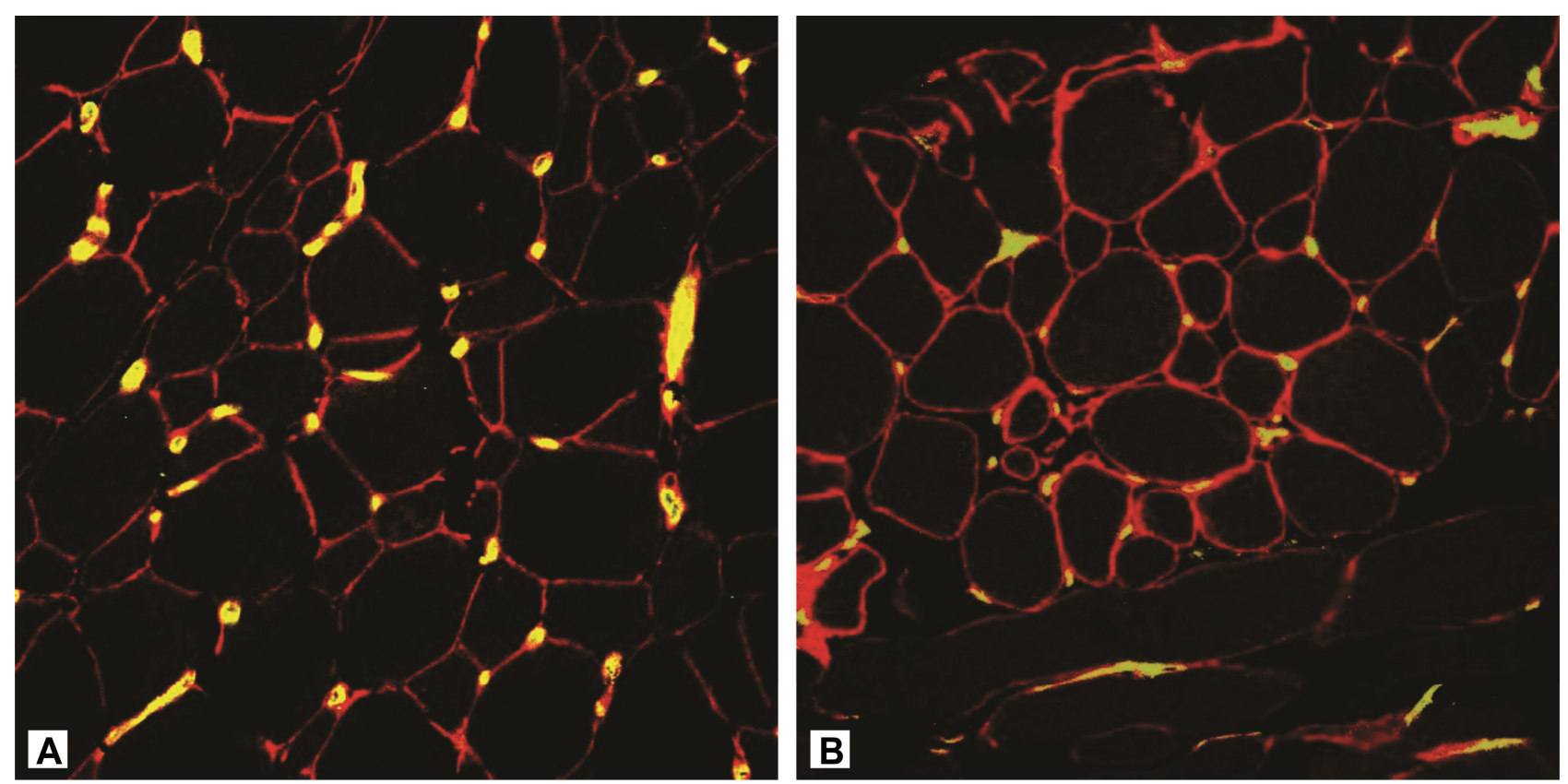

Fig. 1. Capillaries and muscle fibres in human masseter muscle of young (a) and old (b) subject. Merged image of green and red channel. A triple immunofluorescence staining for capillaries (in yellow - green) and muscle fibre outlines (in red). Scale $=100 \mu \mathrm{m}$. 

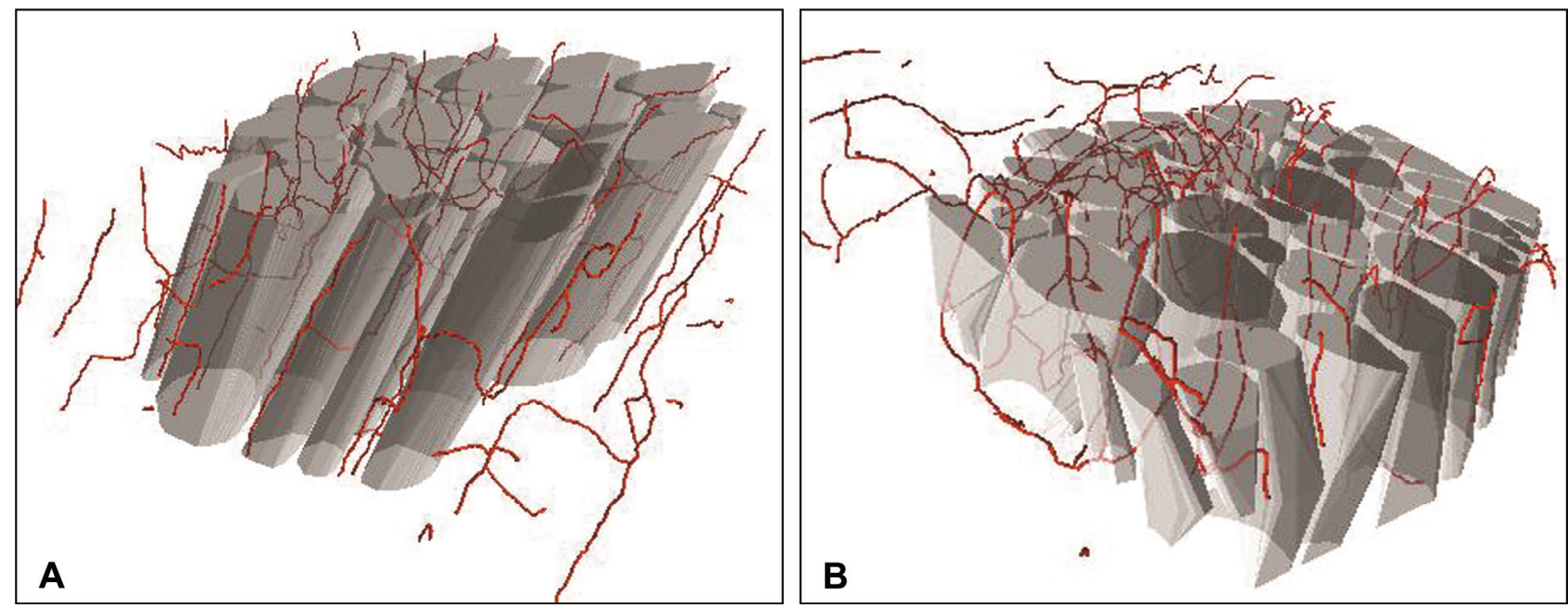

Fig. 2. 3D rendering of capillaries and muscle fibres in masseter muscle of young (a) and old (b) subjects.

Table 1. Capillary pattern in muscle samples of young and old subjects. Network characteristics were estimated by the length of capillaries per muscle volume (Lcap/Vmuscle $\left.\left[\mathrm{\mu m}^{-2}\right]\right) \times 10^{-6}$, tortuosity $\left[\mathrm{rad} \mu \mathrm{m}^{-1}\right] \times 10^{-3}$, anisotropy, number of

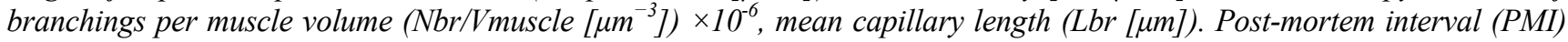
[hours] is indicated for all but one sample.)

\begin{tabular}{ccccccccc}
\hline & Sample & Age & PMI & Lcap/Vmuscle & Tortuosity & Anisotrpy & Lbr & Nbr/Vmuscle \\
\hline Young & 1 & 19.0 & 11 & 448.10 & 78.16 & 1.92 & 158.70 & 1.95 \\
& 2 & 29.0 & 26 & 746.34 & 41.91 & 2.49 & 178.38 & 2.85 \\
& 3 & 29.0 & 21 & 620.99 & 59.04 & 1.76 & 143.48 & 2.92 \\
& 4 & 35.0 & 14 & 629.61 & 80.55 & 2.47 & 189.64 & 2.24 \\
& 5 & 39.0 & 15 & 710.06 & 174.99 & 2.93 & 197.60 & 2.46 \\
& 6 & 19.0 & 25 & 583.39 & 99.94 & 1.75 & 90.27 & 4.47 \\
& 7 & 31.0 & 24 & 655.95 & 97.90 & 1.51 & 76.70 & 5.78 \\
& 8 & 26.0 & 18 & 520.14 & 70.95 & 1.80 & 109.74 & 3.36 \\
\hline Avg & 28.4 & & 614.32 & 87.93 & 2.08 & 143.06 & 3.25 \\
& SD & 6.2 & & 97.15 & 40.02 & 0.49 & 46.20 & 1.28 \\
& SEM & 2.2 & & 34.35 & 14.15 & 0.17 & 16.33 & 0.45 \\
CV & 21.8 & & 15.81 & 45.51 & 23.56 & 32.29 & 39.38 \\
\hline 10 & 73.0 & 21 & 328.14 & 45.34 & 2.40 & 199.34 & 1.13 \\
& 84.0 & 48 & 723.05 & 87.97 & 2.41 & 264.06 & 1.85 \\
& 11 & 83.0 & 15 & 837.92 & 61.77 & 1.71 & 201.96 & 2.77 \\
& 12 & 79.0 & 21 & 429.84 & 54.30 & 1.92 & 161.99 & 2.52 \\
& 13 & 76.0 & 17 & 701.41 & 61.36 & 1.83 & 109.96 & 4.50 \\
& 14 & 84.0 & - & 813.27 & 49.70 & 2.70 & 169.24 & 3.42 \\
& 12 & 77.0 & 24 & 893.68 & 44.00 & 2.68 & 321.26 & 2.13
\end{tabular}

Since most masseter muscles are composed of smaller type 2 and larger type 1 muscle fibres (Cvetko et al., 2012) we estimated capillary characteristics around those two fibre types separately (Figs. 3a,b,c).

Length of capillaries per fibre length (Lcap/Lfib) was larger in type 1 fibre than in type 2 fibres in young subject, but not significantly different in old subjects. With ageing in type 1 fibres Lcap/Lfib dropped significantly $(p=0.041)$ while in type 2 fibres Lcap/Lfib showed tendency to decrease in samples of old subjects, Lcap/Sfib did not change with age, while Lcap/Vfib was significantly smaller in type 1 compared to type 2 fibres $(\mathrm{p}=0.032)$. 

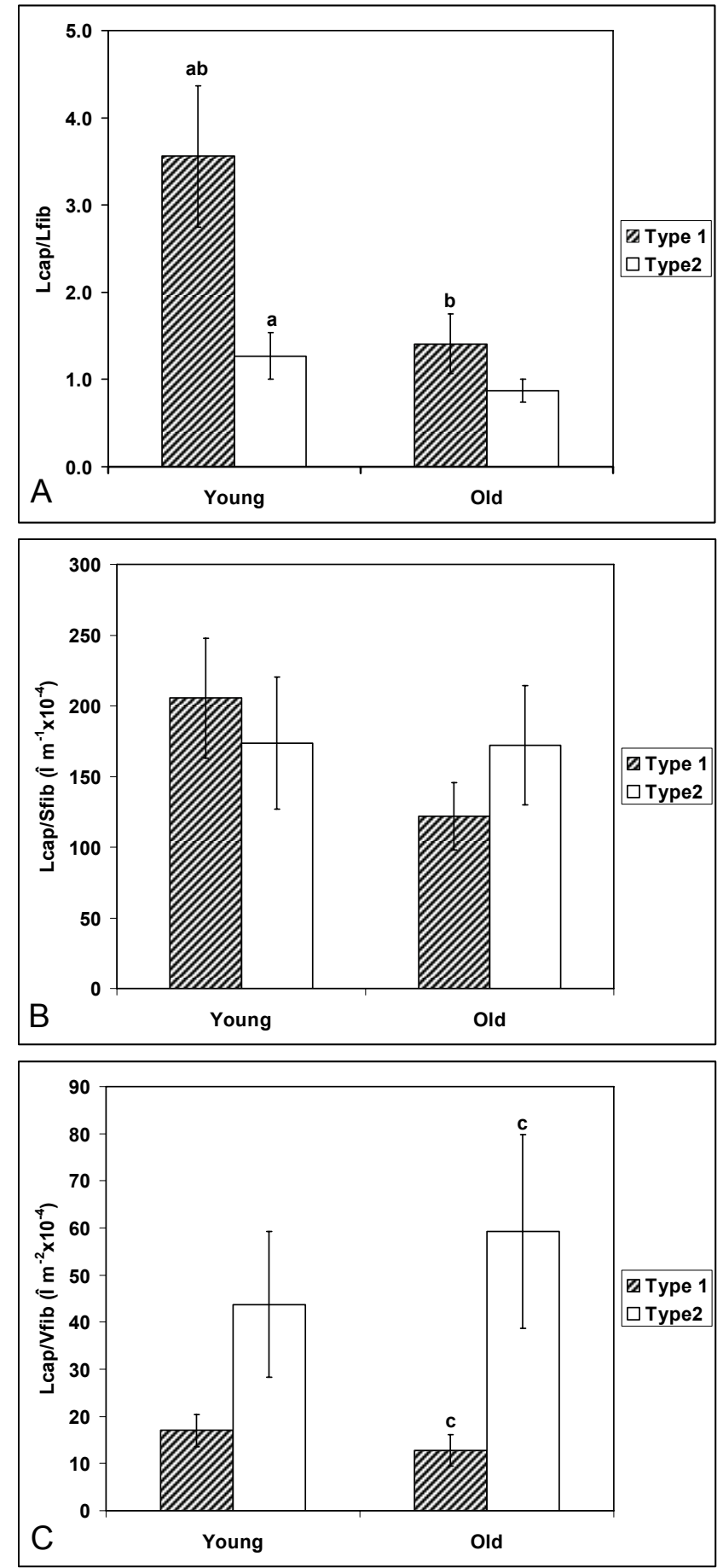

Fig. 3. Capillary supply of muscle fibre types 1 and 2 in young and old human masseter muscles. Network characteristics were estimated by the length of capillaries A) per muscle fibre length (Lcap/Lfib), B) per muscle fibre surface area (Lcap/Sfib $\left[\mu^{-1}\right] \times 10^{-4}$ ) and C) per muscle fibre volume (Lcap/Vfib $\left[\mathrm{\mu m}^{-2}\right]$ $\left.\times 10^{-4}\right),{ }^{a} \mathrm{P}=0.041,{ }^{b} \mathrm{P}=0.026,{ }^{c} \mathrm{P}=0.032$

\section{DISCUSSION}

This study has proved that the length of capillaries per fibre length (Lcap/Lfib) was larger in type 1 fibres than in type 2 fibres in young subjects, but not significantly different in old subjects. With ageing in type 1 fibres Lcap/Lfib dropped significantly, while in type 2 fibres Lcap/Lfib showed just a tendency to decrease, Lcap/Sfib did not change with age, while Lcap/Vfib was significantly smaller in type 1 compared to type 2 fibres. There was a trend toward reduction in curvature and branch density with a consequent elongation of the average capillary branch length in the old group.

The characteristics of the capillary network were quite variable within every age group. Large interindividual variability in capillarity could reflect differences in muscle daily activity and loading pattern due to different craniofacial morphology, intermaxillary relationship and dental status, but also due to methodological problems that could not be totally overcome. All the data were obtained from autopsy material where it is much more difficult to ensure good tissue preservation. Moreover, to our knowledge it is practically impossible to control the extent of autolysis. In Janáček et al. (2011) we describe in detail the methodological problems that needed to be solved before we could get a good staining of capillaries through $100 \mu \mathrm{m}$ muscle section. Further, although UAE lectin is an excellent marker for capillaries in human skeletal muscles, it did not function in several human autopsy muscle samples. Kadota et al. (1986) report that in case of stroke, the time of brain sample excision after stroke is decisive. Maybe, capillaries in skeletal muscles react similarly. We attempted to overcome this problem with additional staining of capillaries with collagen IV and F8. When one or the other marker did not function completely, it was still possible to identify capillaries. In case of complete absence of UAE binding the samples were discarded.

Most muscle samples were excised up to $26 \mathrm{hrs}$ after death and one $48 \mathrm{hrs}$ after death, therefore we assume that post-mortem changes affected all samples in a similar way, though we are well aware that the changes could not be absolutely comparable. We could not find any correlation between values of estimated parameters and the time post mortem. We observed lack of anastomoses especially in the old group. As 
we were not able to find any marker of post-mortem changes - lack of anastomoses could also be ascribed to postmortal degradation and not only to changes due to ageing. Since we could not identify properly the postmortal changes, this is also a potential reason for great variability in our results.

\section{CAPILLARY PATTERN IN MASSETER MUSCLE AT YOUNG AND OLD SUBJECTS}

Data from the present study indicate that there are no significant age-related changes in capillarity of the masseter muscle as a whole; however, larger length of capillaries per unit muscle volume, reduction in curvature and branch density with a consequent elongation of the average capillary branch length point to remodelling of the capillary bed in old subjects. A tendency of larger length of capillaries per unit muscle fibre volume in old masseter muscle reflects smaller diameter of muscle fibres in old subjects. In our previous study in masseter muscle we presented a larger number of branches and greater tortuosity compared to vastus lateralis muscle and to multifidus muscle (Janáček et al., 2011). Curvature and branch density seem to be muscle specific and may represent a compensatory mechanism in masseter muscle to improve diffusive capacity. The trend toward a higher degree of anisotropy, lower tortuosity and branch density found with ageing suggest the age related alteration in geometry in the capillarity in masseter muscle. A tendency toward decreased number of branches - anastomoses among longitudinally oriented capillaries and decreased tortuosity in aged masseter suggest lower metabolic requirements of ageing muscle fibres.

Lower muscle capillarization is accompanied with lower VEGF expression (Croley et al., 2005; Ryan et al., 2006). Angiogenic potential seems not to be disturbed in masseter muscle with age. Although VEGF164 is a predominant form in various tissues, the role of VEGF188 was reported in angiogenesis and capillary maintenance in the masticatory muscles (Ishii et al., 2001). Since anatomical capillarization is well maintained in old masseter muscle, it could be speculated that VEGF188 is not downregulated with age.

Quantitative analysis of capillary supply to skeletal muscle is a prerequisite for understanding the limits to peripheral oxygen transport. This facilitates an increase in the exchange of $\mathrm{O}_{2}$ and nutrients, the resistance to fatigue might thus be improved, especially in the small fast fibres. Consequently the smaller muscle fibres can be advantageous for masseter muscle. This is supported by findings in endurance test studies re- porting that the jaw closing muscles resist fatigue better than arm and hand muscles (van Steenberghe et al., 1978). Further, changes in capillary membrane permeability and differences in enzyme activity of the capillaries might reflect different ability of the muscles to resist fatigue and pain (Sjøgaard et al., 1988).

We confirmed a better capillarization of type 1 than type 2 fibres in young subjects, but not in elder. This is congruent with a $2: 1$ ratio of capillary profiles supplying type 1 compared to type 2 fibres for the anterior portion and 6:1 for the posterior portion in the masseter muscle (Stål et al., 1996) and denser capillary supply of type 1 compared to type 2 fibres regardless age and sex reported in PCA muscle (Lyon et al., 2007).

Lcap/Lfib adjacent to type 1 fibres decreased significantly in masseter muscle in old subjects, which suggests that type 1 muscle fibres are less frequently activated with ageing (altered pattern of muscle use or innervation of type 1 fibres).

Type 1 fibres, which are representative of a slow twitch motor unit, are recruited at relatively low stimulations and are resistant to fatigue (Nordstrom and Miles, 1990). They are probably correlated with the maintenance of the tonic function in the masseter muscle at rest, which characterizes its function. This means that only type 1 fibres would be activated at small force level.

As a result of different daily activity pattern and different loading pattern the need and demand of blood supply to type 1 fibres change with ageing. Changed craniofacial morphology, such as reduced vertical dimension of the face and reduced weight of the mandible (teeth loss followed by bone resorption) impose reduced stretch on muscle fibres followed by reduced activity of type 1 fibres. Reduced metabolic needs of type 1 fibres provoke adjustment of capillary network adjacent to type 1 fibres. An age-associated decrease of type 1 fibres and increase in proportion of type 2 fibres were demonstrated in masseter muscle (Cvetko et al., 2012). This data support a shift toward the less oxidative muscle phenotype. The age-related changes in terms of craniofacial morphology are paralleled by alterations in muscle fibre type composition (transitions to fast phenotype) and adjustment of capillarization adjacent to type 1 fibres.

Changes in the ratio of fibre types in jaw muscles based on MyHC expression could be due to age-related changes in neural or hormonal influences (Korfage et al., 2005) and changed muscle use (Basu et al., 2002). Muscular changes due to inactivity often occurring in 
limb muscles do not happen in jaw muscles since habitual oral motor movements (chewing, talking, and swallowing) are maintained in elderly subjects. An important functional consequence of the observed apparent shift towards faster, therefore more powerful fibre types is that this shift may compensate for the loss of muscle power due to reduced mass in the old age.

Similarly to decreased proportion of type 1 fibres with age in masseter muscle data from human laryngeal thyroarythenoid muscle show that there is an age-related loss of type 1 fibres. This suggests that there is a combination of fibre transformations with a subsequent reorganization of the vasculature.

In young subjects the length of capillaries per fibre length (Lcap/Lfib) was significantly larger in capillaries adjacent to type 1 fibres than to type 2 fibres. Greater Lcap/Lfib in type 1 fibres compared to type 2 fibres is in concordance to findings that fast to slow fibre remodelling of the masticatory muscles also encompasses an increase in capillary density (Gedrange et al., 2003), the capillary length supplying the fibre unit volume (Lcap/Vfib) is larger for type 2 fibres, i.e. the unit fibre volume, supplied by the unit capillary length is smaller in type 2 muscle fibres. This probably reflects the metabolic needs of type 2 fibres with predominantly glycolytic metabolism.

Our findings show that anatomic capillarity is well maintained in masseter muscle in old subjects. We conclude that alterations in the anatomic size of the capillary/fibre interface do not play a major role in the fall in skeletal muscle aerobic performance with ageing.

\section{ACKNOWLEDGEMENTS}

The present study was supported by the Slovenian Research Agency (P3-043) and by the public funds provided by the Academy of Sciences of the Czech Republic (AV0Z50110509 and RVO:67985823). We are grateful to Mrs. Nataša Pollak, Mrs. Majda ČrnakMaasarani, Mrs. Andreja Vidmar, Mr. Ivan Blažinovič, Mr. Marko Slak and Mr. Milan Števanec for their skilful technical assistance.

\section{REFERENCES}

Basu R, Basu A, Nair KS (2002). Muscle changes in aging. J Nutr Health Aging 6:336-41.

Brown M (1987). Change in fiber size, not number, in ageing skeletal muscle. Age Ageing 16:244-8.

Brown M, Cotter Ma, Hudlická O, Vrbová G (1976). The effects of different patterns of muscle activity on capillary density, mechanical properties and structure of slow and fast rabbit muscles. Pflugers Arch 361:241-50.

Coggan AR, Spina RJ, King DS, Rogers MA, Brown M, Nemeth PM, Holloszy JO (1992). Histochemical and enzymatic comparison of the gastrocnemius muscle of young and elderly men and women. J Gerontol 47: B71-6.

Cotter M, Hudlická O, Pette D, Staudte H, Vrbová G (1973). Changes of capillary density and enzyme pattern in fast rabbit muscles during long-term stimulation. J Physiol-London 230:34P-35P.

Croley AN, Zwetsloot KA, Westerkamp LM, Ryan NA, Pendergast AM, Hickner RC, et al. (2005). Lower capillarization, VEGF protein, and VEGF mRNA response to acute exercise in the vastus ateralis muscle of aged vs. young women. J Appl Physiol 99:1872-9.

Cvetko E, Karen P, Janáček J, Kubínová L, Plasencia AL, Eržen I (2012). Human masseter muscle fibres from the elderly express less neonatal myosin than those of young adults. Anat Rec (Hoboken) 295:1364-72.

Cui L, Ju Y, Ding L, Trejo-Morales M, Olfert IM (2008). Arteriolar and venular capillary distribution in skeletal muscles of old rats. Gerontol A Biol Sci Med Sci 63: 928-35.

Čebašek V, Eržen I, Vyhnal A, Janáček J, Ribarič S, Kubínová L (2010). The estimation error of skeletal muscle capillary supply is significantly reduced by $3 \mathrm{D}$ method. Microvasc Res 79:40-6.

Degens H, Turek Z, Hoofd LJC, Binkhorst RA (1994). Capillary proliferation related to fibre types in hypertrophied ageing rat m. plantaris. Adv Exp Med Biol 345:669-76.

Denis C, Chatard JC, Dormois D, Linossier MT, Geyssant A, Lacour JR (1986). Effects of endurance training on capillary supply of human skeletal muscle on two age groups (20 and 60 years). J Physiol (Paris) 81:379-83.

Dorph-Petersen KA, Nyengaard JR, Gundersen HJG (2001). Tissue shrinkage and unbiased stereological estimation of particle number and size. J Microsc 204:232-46.

Egginton S, Hudlická O (2000). Selective long-term electrical stimulation of fast glycolytic fibres increases capillary supply but not oxidative enzyme activity in rat skeletal muscles. Exp Physiol 85:567-73.

Eržen I, Janáček J, Kubínová L (2011). Characterization of the capillary network in skeletal muscles from 3D data. Physiol Res 60:1-13.

Frontera WR, Hughes VA, Fielding RA, Fiatarone MA, Evans WJ, Roubenhoff R (2000). Aging of skeletal muscle: a 12-year longitudinal study. J Appl Physiol 88:1321-6.

Gambino DR, Malmgren LT, Gacek RR (1990). Agerelated changes in the neuromuscular junctions in the human posterior cricoarytenoid muscles: a quantitative study. Laryngoscope 100: 262-8.

Gedrange T, Walter B, Tetzlaff I, Kasper M, Schubert H, Harzer W, et al. (2003). Regional alterations in fiber type distribution, capillary density, and blood flow after 
lower jaw sagittal advancement in pig masticatory muscles. J Dent Res 82:570-4.

Gundersen HJG, Boyce RW, Nyengaard JR, Odgaard A (1993) The Conneulor: unbiased estimation of connectivity using physical disectors under projection. Bone 14:217-22.

Hell S, Reiner G, Cremer C, Stelzer EHK (1993) Aberrations in confocal fluorescence microscopy induced by mismatches in refractive index. Microsc 169:391-405.

Hepple RT, Vogell JE (2004). Anatomic capillarization is maintained in relative excess of fibre oxidative capacity in some skeletal muscles of late middle-aged rats. J Appl Physiol 96:2257-64.

Ishii H, Oota I, Takuma T, Inomata K (2001). Developmental expression of vascular endothelial growth factor in the masseter muscle of rats. Arch Oral Biol 46:77-82.

Janáček J, Cvetko E, Kubínová L, Travnik L, Eržen I (2011). A novel method for evaluation of capillarity in human skeletal muscles from confocal 3D images. Microvasc Res 81:231-8.

Janáček J, Kreft M, Čebašek V, Eržen I (2012). Correcting the axial shrinkage of skeletal muscle thick sections visualized by confocal microscopy. J Microsc 246: 107-2.

Kadota E, Tanji K, Nishida S, Takahashi M, Maeda M, Hiruma S, et al. (1986). Lectin (UEA -1) reaction of capillary endothelium with reference to permeability in autopsied cases of cerebral infarction. Histol Histopathol 1:219-26.

Knust J, Ochs M, Gundersen HJG, Nyengaard JR (2009). Stereological estimates of alveolar number and size and capillary length and surface area in mice lungs. Anat Rec 292:113-22.

Korfage JA, Koolstra JH, Langenbach GE, van Eijden TM (2005). Fiber-type composition of the human jaw muscles (part 1) origin and functional significance of fiber-type diversity. J Dent Res 84:774-83.

Kubínová L, Janáček J, Ribarič S, Čebašek V, Eržen I (2001). Three-dimensional study of the capillary supply of skeletal muscle fibres using confocal microscopy. J Muscle Res Cell Motil 22:217-27.

Lyon MJ, Steer LM, Malmgren LT (2007). Stereological estimates indicate that aging does not alter the capillary length density in the human posterior cricoarytenoid muscle. J Appl Physiol 103:1815-23.

Mathieu-Costello O, Ju Y, Trejo-Morales M, Cui L (2005). Greater capillary-fibre interface per fibre mitochondrial volume in skeletal muscles of old rats. J Appl Physiol 99:281-9.

Mitchell ML, Byrnes WC, Mazzeo RS (1991). A comparison of skeletal muscle morphology with training between young and old Fischer 344 rats. Mech Ageing Dev 58: 21-35.

Nelissen-Vrancken HJ, Boudier HA, Daemen MJ, Smits JF (1993). Antihypertensive therapy and adaptive mechanisms in peripheral ischemia. Hypertension 22:780-8.

Nordstrom MA, Miles TS (1990). Fatigue of single motor units in human masseter. J Appl Physiol 68:26-34.

Nyengaard JR (1999). Stereologic methods and their application in kidney research (Review). J Am Soc Nephrol 10:1100-23.

Palágyi K, Kuba A (1998). A 3D 6-subiteration thinning algorithm for extracting medial lines. Pattern Rec Lett 19:613-27.

Proctor DN, Sinning WE, Walro JM, Sieck GC, Lemon PW (1995). Oxidative capacity of human muscle fiber types: effects of age and training status. J Appl Physiol 78:2033-8.

Ryan NA, Zwetsloot KA, Westerkamp LM, Hickner RC, Pofahl WE, Gavin TP (2006). Lower skeletal muscle capillarization and VEGF expression in aged vs. young men. J Appl Physiol 100:178-85.

Sjøgaard G (1982). Capillary supply and cross-sectional area of slow and fast twitch muscle fibres in man. Histochemistry 76:547-55.

Sjøgaard G, Savard G, Juel C (1988). Muscle blood flow during isometric activity and its relation to muscle fatigue. Eur J Appl Physiol Occup Physiol 57:327-35.

Stål P, Eriksson PO, Eriksson A, Thornell LE (1990). Enzyme-histochemical and morphological characteristics of muscle fibre types in the human buccinator and orbicularis oris. Arch Oral Biol 35:449-58.

Stål P, Eriksson PO, Thornell LE (1996). Differences in capillary supply between human oro-facial, masticatory and limb muscles. J Muscle Res Cell Motil 17:183-97.

Škorjanc D, Jaschinski F, Heine G, Pette D (1998). Sequential increases in capillarization and mitochondrial enzymes in low-frequency-stimulated rabbit muscle. Am J Physiol Cell Physiol 274:C810-8.

Štencel M, Janáček J (2006). On calculation of chamfer distance and Lipschitz covers in digital images. Proceedings S4G. Lechnerová R, Saxl I, Beneš V, eds. Prague, Union of Czech Mathematicians and Physicists, 517-22.

van Steenberghe D, De Vries JH, Hollander AP (1978). Resistance of jaw-closing muscles to fatigue during repetitive maximal voluntary clenching efforts in man. Arch Oral Biol 23:697-701.

Wulfson D, Knust J, Ochs M, Nyengaard JR, Gundersen HJG (2009) Stereological estimation of the total number of ventilator units in mice lungs. J Microsc 238: 75-89. 